\title{
IMPLEMENTACIÓN DE PERSONAL RESPONSE SYSTEMS EN ASIGNATURAS DE LOS GRADOS DE QUÍMICA Y BIOQUÍMICA
}

\section{IMPLEMENTATION OF PERSONAL RESPONSE SYSTEMS IN SUBJECTS OF THE DEGREES OF CHEMISTRY AND BIOCHEMISTRY}

\author{
José Ángel Salatti-Dorado, Beatriz María Fresco Cala, \\ María Isabel López Martínez, Julia Ríos Gómez, \\ Mª Soledad Cárdenas Aranzana*, Juan Manuel Fernández Romero, \\ Francisco José Romero Salguero \\ Facultad de Ciencias, Universidad de Córdoba. 14072, Córdoba, SPAIN \\ *scardenas@uco.es
}

\begin{abstract}
Resumen
La enseñanza en la Universidad pretende desarrollar capacidades de autoaprendizaje, de iniciativa y capacidad de visualización de los problemas planteados en cualquiera de sus ámbitos profesionales, así como la habilidad de adoptar soluciones que permitan resolver los problemas que la sociedad les plantee. Por otra parte, la innovación es un concepto que debe ir incorporándose transversalmente a la docencia, es por ello que cada vez más habitualmente se están usando herramientas que estimulan la participación activa de los alumnos. Por ello, los Personal Response Systems (PRS) permiten al alumno establecer un feedback con el docente mediante un dispositivo electrónico donde se realiza una serie de preguntas y los resultados se obtienen e interpretan en tiempo real. Se ha pretendido con este artículo de innovación docente disponer de una herramienta que permita conocer en tiempo real y de una forma simple si el alumno es capaz de responder a cuestiones sencillas, con un marcado carácter aplicado para evaluar el grado de comprensión de la materia. Este artículo se ha basado en la realización de una serie de actividades englobadas en una actividad central basada en el estudio de casos reales relacionados con diferentes temáticas El cuestionario se aplicó en asignaturas del Grado de Química y Bioquímica en las preguntas se realizaron sobre aspectos teóricos y prácticos de las mismas, así como sobre exposiciones preparadas por los alumnos. También se introdujo el idioma en el que se impartían (castellano e inglés) como variable adicional para la evaluación de los resultados obtenidos en los diferentes grupos de docencia.
\end{abstract}

Palabras clave: Personal Response System (PRS), Kahoot

\section{Abstract}

Nowadays the teaching context is undergoing changes in the teaching-learning binomial that require a more active participation by the students for a better assimilation of knowledge, skills and competences. On the other hand, it is also necessary that the student knows how to apply this knowledge to practical cases, for which it is necessary to adapt the conventional methodologies so that custom work by students is encouraged.

It is intended with this teaching innovation investigation to use Personal Response Systems (PRS) as a tool that allows to know in real time and in a simple way if the students are able to answer simple questions, with a marked applied character, to evaluate the degree of understanding of the subject. In this sense, the use of new technologies, specifically the mobile applications, seems to be a friendly environment for the students that will predispose them favorably to the development of this activity. Since it is also a question of facilitating the teaching initiation of the four young teachers who make up this proposal, they will be in charge of the design of this teaching material and its subsequent evaluation supervised by the tutors of the group. The introduction of PRS in a subject can imply a new approach with very positive connotations for both the students and the teachers. The teaching innovation work will be applied in the subjects Instrumental Analysis I, Bioanalytical Chemistry and Bioorganic Chemistry, of the degrees of Chemistry and Biochemistry.

Keywords: Personal Response Systems (PRS); Kahoot.

\section{INTRODUCCIÓN}

La enseñanza en la Universidad pretende, entre otros objetivos, desarrollar capacidades de autoaprendizaje, iniciativa, y capacidad de visualización de los problemas planteados en cualquiera de sus ámbitos profesionales, así como la capacidad de adoptar soluciones que permitan resolver los problemas que la sociedad plantea. El contexto docente actual ha implicado un cambio en la forma en la que el profesor y el estudiante se enfrentan a la explicación y al estudio, respectivamente, de una materia [1]. El proceso de Convergencia Europea y el consabido binomio enseñanza-aprendizaje conllevan una participación mucho más activa del alumno en el proceso de adquisición de conocimientos, habilidades y competencias que le permita alcanzar una formación integral, la cual le facilite su inserción laboral. En este sentido, se requiere que el alumno aplique los 
conocimientos adquiridos en las clases magistrales a supuestos prácticos. Por parte del profesor, esto implica una adaptación de la metodología convirtiéndose en supervisor/tutor e incrementando el protagonismo del alumno. Por ello, cada día es más habitual la incorporación de herramientas interactivas que estimulan la participación activa de los estudiantes.

Los Personal Response Systems (PRS) son una herramienta tecnológica que facilita al docente interactuar con todos los estudiantes en un aula de manera concurrente utilizando un medio electrónico; los estudiantes responden a través de un dispositivo que cada uno posee (móvil o Tablet) a preguntas que el docente hace a través de un medio visual, por ejemplo una plataforma virtual [2].

La tecnología emplea un sistema basado en hardware, un control remoto (uno por cada persona en el aula), y un receptor que permite la captura de la información enviada utilizando el control. Finalmente el software permite conocer los resultados de la información recogida [2].

El docente puede realizar evaluaciones y sondeos con el fin de determinar el grado de comprensión de lo que se está explicando. Además, los resultados se pueden obtener al instante, pudiendo incidir en aquellos aspectos menos asimilados por los alumnos. El anonimato en las encuestas facilita la participación del estudiante. Además, la visualización de los participantes que adquieren mayor puntuación motiva a los estudiantes manteniendo la concentración. Una vez finalizada la actividad generan un cuestionario de satisfacción para conocer, a tiempo real, la opinión y la valoración de los estudiantes. Sin embargo, la mayor desventaja que presenta este tipo de dispositivos es la necesidad de desarrollar un software específico y del conocimiento necesario por parte del profesor para su correcto funcionamiento.

En 2013 aparece Kahoot, una página web de acceso libre que permite desarrollar la actividad descrita anteriormente, pero con algunas ventajas añadidas: la realización de cuestionarios online a través de dispositivos electrónicos con acceso a internet como los smartphones, tablets u ordenador [3].

A lo largo de este curso académico (2016-2017) se ha usado dicha herramienta para el seguimiento de los conocimientos adquiridos por diferentes grupos de alumnos pertenecientes a distintos niveles y asignaturas. Este sistema ha permitido la comparación de los resultados obtenidos tanto dentro de una misma clase como entre diferentes tipos de docencia presencial (prácticas de laboratorio y/o seminarios teóricos y prácticos), en grupos de docencia en castellano e inglés.

\section{OBJETIVOS}

- Introducir los Personal Response Systems en las asignaturas Análisis Instrumental I, Química Bioanalítica y Química Bioorgánica.

- Evaluar su utilidad como herramienta de preevaluación/ autoevaluación de los estudiantes, sin repercusiones académicas.

- Preparar modelos de cuestionarios basados en situaciones reales que pudiesen extenderse a otras asignaturas.

- Formar al profesorado novel en la preparación de este tipo de actividades para su posterior aplicación.

\section{Metodología}

Para la ejecución de este trabajo se propuso una metodología general que ha sido el hilo conductor del mismo. En concreto, el plan de trabajo a seguir establece una serie de pautas, que se van uniendo y autoalimentandose a lo largo de período de duración de cada asignatura. El plan de trabajo se concretó en los siguientes aspectos:

1. Búsqueda de los supuestos prácticos que serían objeto de las preguntas de los cuestionarios.

2. Realización de una serie de cuestionarios convencionales llevados a cabo en diferentes sesiones conforme se haya impartiendo la materia. Dichas sesiones fomentaron el trabajo individual, siendo de esta manera sesiones preparativas de las siguientes actividades programadas.

3. Puesta en práctica de nuevas estrategias de participación del alumnado. Consistió en una sesión final donde se usó la herramienta Kahoot para la realización de un cuestionario online a tiempo real donde todos los alumnos participaron individual y activamente. Las preguntas de dicho cuestionario se extrajeron en un porcentaje significativo de los cuestionarios realizados durante la primera etapa.

4. Evaluación de los resultados del aprendizaje y comparación de estos con los del nivel alcanzado por cada estudiante para detectar mejoras en aquellos aspectos que se hayan identificado como deficientes en las PRS.

\section{- DESCRIPCIÓN}

Puesto que se trata también de facilitar la iniciación docente de los cuatro profesores noveles, han sido los encargados del diseño de este material docente y su posterior evaluación supervisada por los profesores tutores del grupo. La 
introducción de los PRS en estas asignaturas puede suponer un nuevo enfoque con connotaciones muy positivas tanto para el alumno como para los profesores.

La realización de la evaluación en este trabajo ha sido a través de la aplicación "Kahoot", un sistema de evaluación mediante cuestionarios con varias opciones de respuesta que aparecen en los dispositivos de cada alumno pudiendo contestar de forma independiente e instantánea. Esta aplicación funciona mediante la conexión en red local con los dispositivos tecnológicos de forma simultánea.

El trabajo de innovación docente se ha aplicado en las asignaturas Análisis Instrumental I del Grado de Química y Química Bioorgánica y Química Bioanalítica, ambas del Grado de Bioquímica.

\subsection{ANÁLISIS INSTRUMENTAL I}

La asignatura Análisis Instrumental I se imparte en el primer cuatrimestre del tercer curso del grado de Química, y tiene carácter obligatorio. Dicha asignatura supone la toma de contacto de los alumnos con las técnicas ópticas, conjugando la explicación de los fundamentos teóricos de dichas técnicas con la descripción más o menos pormenorizada de los elementos que constituyen los instrumentos de medida, así como las configuraciones de estos. De esta manera, se persigue que el alumno conozca sus capacidades y limitaciones y adquiera una visión de su utilidad actual y de su potencial, siendo capaz de discernir la técnica adecuada para la resolución de problemas analíticos concretos.

En este caso, la aplicación de este estudio de innovación docente se ha centrado en la respuesta a preguntas de respuesta breve relacionadas con contenidos teóricos de la materia. La realización del cuestionario Kahoot se ha llevado a cabo en dos sesiones distintas. En la primera, las cuestiones estuvieron relacionadas con las técnicas ópticas moleculares y en la segunda, con técnicas ópticas atómicas. Las cuestiones tenían 4 opciones entre las cuales solamente una podía ser la correcta, teniendo un tiempo máximo de 10 segundos por cuestión para responder.

\subsection{QUÍMICA BIOANALÍTICA}

La asignatura Química Bioanalítica se imparte en el primer cuatrimestre del cuarto curso del grado de Bioquímica. Dicha asignatura se compone de una serie de actividades formativas las cuales van desde la realización de seminarios como la lectura de textos científicos o el análisis de casos concretos. Concretamente, el trabajo se realizó en la lección 3 de la misma y en las Prácticas de aula PA2.

Cada cuestión tuvo 4 opciones de respuesta, de las cuales solamente una puede ser la correcta teniendo un tiempo máximo de 10 segundos para responderlas. La realización de este cuestionario se hizo el mismo día de exposición de la PA2 y estuvo compuesto por 8 preguntas generales de la lección 3, y 7 preguntas específicas relativas de cada uno de los temas expuestos por los alumnos.

Una vez realizado el cuestionario y el estudio de los resultados obtenidos, se procedió a presentar la discusión de los mismos en una clase posterior.

\subsection{QUíMICA BIOORGÁNICA}

La asignatura Química Bioorgánica es una asignatura de carácter optativo y pertenece, al igual que la asignatura anterior, al grado de Bioquímica. Concretamente, se imparte en el primer cuatrimestre del cuarto curso y se compone tanto de contenidos teóricos como de contenidos prácticos. Respecto al contenido práctico, destaca el aprendizaje del acceso y manejo de bases de datos bibliográficas de interés en Química Orgánica y Bioquímica y realización de prácticas de laboratorio.

Con esta asignatura se pretende que los alumnos conozcan algunas familias de compuestos orgánicos de interés en Bioquímica, entiendan los principios básicos de la biocatálisis y sepan aplicar métodos y técnicas de la Química Orgánica para resolver problemas biológicos.

Esta asignatura se imparte tanto en español como en inglés. La evaluación de esta asignatura con Kahoot se llevó a cabo en la parte práctica de la asignatura impartida en inglés.

\section{RESULTADOS OBTENIDOS}

A continuación, se van a mostrar los resultados obtenidos en cada uno de los cuestionarios realizados en cada una de las asignaturas citadas anteriormente.

\subsection{ANÁLISIS INSTRUMENTAL I}

Tras la realización de la evaluación se obtuvieron los resultados que se muestran en la Figura 1. Como puede observarse, el porcentaje de respuestas acertadas en cada cuestionario entre subgrupos son similares, rondando el $60 \%$ de 
aciertos. Tampoco, hubo diferencias significativas entre las dos partes de las asignaturas (técnicas atómicas y moleculares). Esto permite evaluar la comprensión y asimilación por parte del alumnado de los conceptos teóricos explicados previamente en clase.
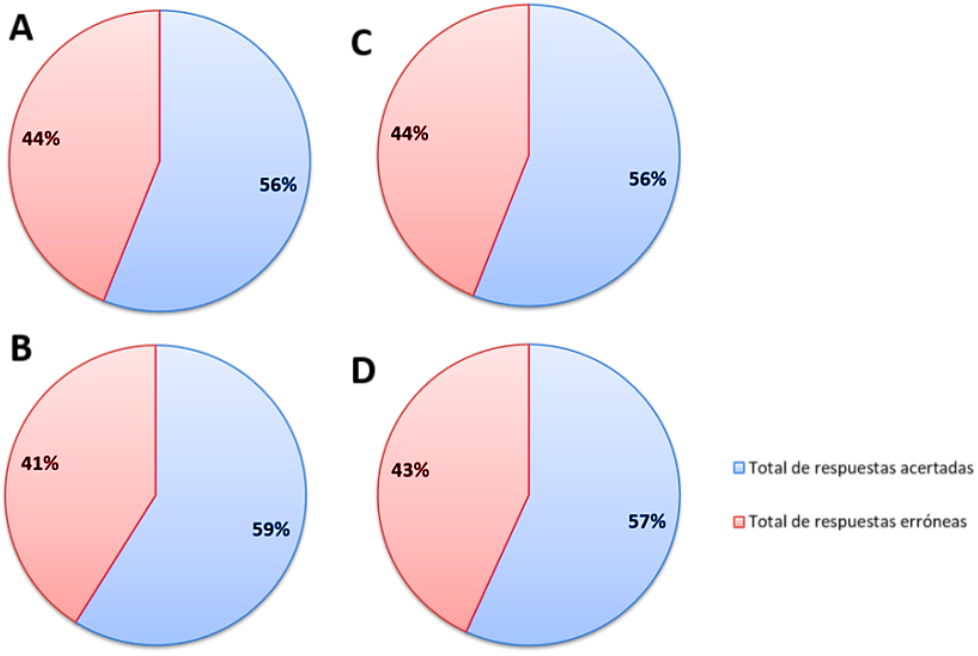

Figura 1. Relación de respuestas acertadas y erróneas del cuestionario sobre técnicas atómicas; subgrupo 1 (A) y 2 (B), y del cuestionario sobre técnicas moleculares; subgrupo 1 (C) y 2 (D).

\subsection{QUÍMICA BIOANALÍTICA}

Una vez realizada la evaluación en la asignatura Química Bioanalítica, se obtuvieron los resultados plasmados en la Figura 2. Como puede observarse, la tasa de éxito en las respuestas es el 66\%. Además, hemos podido determinar que un $60 \%$ de los alumnos que realizaron la evaluación obtuvieron un mayor número de respuestas acertadas que erróneas $(>8$ respuestas acertadas) lo que nos permite determinar el grado de éxito en la explicación de la parte teórica implicada en dicho test, así como el alto grado de atención que los alumnos mostraron en los seminarios realizados por sus compañeros.

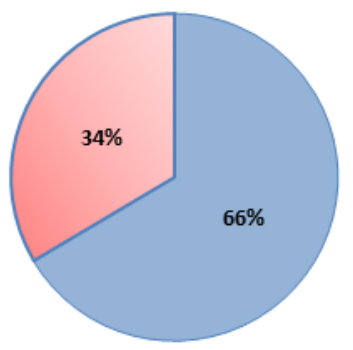

\section{Total de respuestas acertadas}

$\square$ Total de respuestas erróneas

Figura 2. Relación de respuestas acertadas y erróneas en el cuestionario de la asignatura de Química Bioanalítica.

\subsection{QUímICA BIOORGÁNICA}

Tras la realización de las prácticas de laboratorio, se procedió a la evaluación de las mismas utilizando la herramienta Kahoot. Los resultados obtenidos se muestran en la Figura 3. 


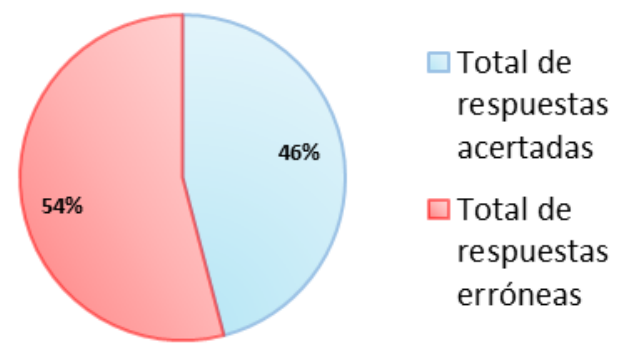

Figura 3. Relación de respuestas acertadas y erróneas del cuestionario sobre las prácticas de laboratorio de la asignatura Química Bioorgánica.

A diferencia de los resultados obtenidos en los cuestionarios realizados en las otras dos asignaturas, el porcentaje de respuestas acertadas no superó el 50\%. Esto se ha podido deber a la dificultad añadida que supone la realización del cuestionario en inglés.

\section{UTILIDAD/ANÁLISIS}

El cuestionario de satisfacción de la actividad, cuyos resultados pueden verse en la Tabla 1, nos muestra que una media del $76.05 \%$ de los alumnos encontraron este tipo de actividad positiva para su aprendizaje; La aplicación de Kahoot ha resultado ser una actividad amena para un promedio de $84.75 \%$ de los alumnos de las tres asignaturas. Una media del $92.77 \%$ de los alumnos recomendó esta aplicación como un método alternativo de aprendizaje, siendo un $86.87 \%$ de los mismos los que consideran dicha herramienta útil a la hora de incorporar y reforzar conocimientos.

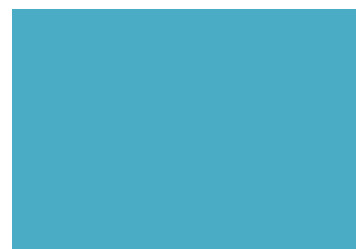

\begin{tabular}{|c|c|c|c|}
\hline \multicolumn{4}{|c|}{ ASIGNATURAS } \\
\hline \multicolumn{2}{|c|}{$\begin{array}{l}\text { Análisis Instrumental } \\
\text { I }\end{array}$} & \multirow{2}{*}{$\begin{array}{c}\text { Química } \\
\text { Bioanalítica }\end{array}$} & \multirow{2}{*}{$\begin{array}{l}\text { Química } \\
\text { Bioórganica }\end{array}$} \\
\hline $\begin{array}{l}\text { Ténicas } \\
\text { atómicas }\end{array}$ & $\begin{array}{c}\text { Técnicas } \\
\text { moleculares }\end{array}$ & & \\
\hline 81.8 & 85.6 & 93.4 & 78.2 \\
\hline 86.0 & 96.3 & 89.0 & 76.2 \\
\hline 93.0 & 96.3 & 96.8 & 85.0 \\
\hline 66.7 & 75.9 & 83.3 & 78.3 \\
\hline 27.8 & 20.7 & 8.3 & 21.7 \\
\hline 5.5 & 3.4 & 8.3 & 0.0 \\
\hline
\end{tabular}

Tabla 1. Resultados cuestionario de satisfacción para cada una de las asignaturas implicadas en el estudio. 


\section{CONCLUSIONES/DISCUSIÓN}

El análisis de los resultados obtenidos en la presente investigación de innovación docente nos ha permitido poner de manifiesto la utilidad de este tipo de aplicaciones interactivas, que fomentan la participación del estudiante.

El modelo se ha aplicado a diferentes asignaturas de características educativas muy variadas tanto en el tipo de alumnos (estudiantes de los grados de Química y Bioquímica de diferentes cursos), la lengua utilizada para la impartición de la misma (inglés y español) y para cualquier número de alumnos. Esto ha sido posible dada la versatilidad de la aplicación móvil seleccionada que permite el diseño ad-hoc del cuestionario.

La herramienta también ha sido útil para evaluar el nivel de aprendizaje y atención de los alumnos por parte del profesor. Asimismo, el alumno ha podido autoevaluarse para determinar el grado real de conocimiento adquirido durante el desarrollo de las asignaturas.

En nuestra opinión, este tipo de experiencia, no sólo sirve como herramienta de trabajo para los docentes, sino que, además permite al alumno acercarse de una manera más amena a aspectos más aplicados de los conocimientos que cada asignatura puede mostrar.

\section{BIBLIOGRAFÍA}

[1] Pérez Miriam González, "Evaluación Del Aprendizaje En La Enseñanza Universitaria,” Rev. Pedagog. Univ., vol. 5, no. 2, pp. 31-55, 2000.

[2] J. S. Herrera, "Innovación docente y uso de las TIC en la enseñanza universitaria," Rev. Univ. y Soc. del ...., vol. 1 , no. 1, pp. 1-16, 2004.

[3] "What is Kahoot?" 